ARTí́CULO

\title{
Nuevos registros y lista actualizada de las algas pardas (Phaeophyceae) del litoral de Michoacán, México
}

\author{
New records and updated list of brown algae (Phaeophyceae) from the coast of \\ Michoacán, Mexico \\ Ángela Catalina Mendoza-González ${ }^{1 *}$, Luz Elena Mateo-Cid ${ }^{1}$, María del Rosario \\ Ortega-Murillo², Leslie Zurita-Valencia ${ }^{2}$, Juan Diego Sánchez-Heredia' ${ }^{2}$ Cynthia \\ Mariana Hernández-Casas ${ }^{1}$
}

\author{
${ }^{1}$ Departamento de Botánica, Escuela Nacional de Ciencias Biológicas, Instituto Politécnico Nacional, Carpio y Plan de Ayala s/n, \\ Colonia Santo Tomás, 11340 Ciudad de México, México \\ ${ }^{2}$ Facultad de Biología, Universidad Michoacana de San Nicolás de Hidalgo, Gral. Francisco J. Múgica s/n, Ciudad Universitaria, \\ 58030 Morelia, Michoacán, México \\ *Autor corresponsal: am7124@gmail.com
}

\begin{abstract}
Brown algae (Phaeophyceae) recorded in the literature and specimens at herbaria from the littoral of Michoacán were extensively reviewed. Additionally, these data were supplemented with samplings in 11 localities from 2006 to 2014 . A total of 52 infrageneric taxa of brown algae (49 species and 3 varieties) were determined; from these, 27 were new records for Michoacán, being Streblonema anomalum and Ectocarpus taoniae new records for the Pacific coast of Mexico. The highest number of species was recorded for La Majahuita (27), while the lowest number of taxa was in San Telmo (2). The family Dictyotaceae was the best represented (15 taxa), followed by Chordariaceae and Ectocarpaceae ( 5 each one). From the previous 25 records in this area, the presence of 12 taxa was confirmed, so the number of Phaeophyceae from this coast increased to 52 taxa. The floristic list is accompanied by data of presence, seasonality, reproduction, habitat and bibliographic references. Species diversity was compared between the dry and rainy seasons during 2006 to 2014, the greatest diversity was found during the dry season. The coast of Michoacán has the highest specific richness of Phaeophyceae when comparing to Nayarit, Colima, Jalisco, Guerrero, Oaxaca and Chiapas.
\end{abstract}

Key words: Phaeophyceae; inventory, seasonality, species richness

Resumen.- Se realizó una investigación bibliográfica y de herbario de Phaeophyceae citada para el litoral de Michoacán, México. Se añadieron datos obtenidos de muestreos realizados en 11 localidades de 2006 a 2014, con el objetivo de contribuir al conocimiento de las Phaeophyceae de la región. Se determinaron 52 taxones (49 especies y 3 variedades), 27 son nuevos registros para Michoacán, se citan por primera vez: Streblonema anomalum y Ectocarpus taoniae para la costa del Pacífico de México. El número más alto de taxones se registró en La Majahuita (27) y el más bajo en San Telmo (2). Las familias mejor representadas fueron Dictyotaceae (15 taxones) seguidas por Chordariaceae y Ectocarpaceae (5 cada una). De 25 especies registradas en la bibliografía, se corroboró la presencia de 12. Con los nuevos registros el número de feofíceas se incrementó a 52 taxones. La lista florística se acompaña con datos de presencia, estacionalidad, estado fenológico, hábitat y referencias bibliográficas. Se comparó la riqueza específica entre las estaciones climáticas de lluvias y de secas durante el periodo de estudio, encontrándose el número más alto de taxones en la época de secas. El litoral de Michoacán tiene la riqueza específica más alta de Phaeophyceae, al compararse con Nayarit, Colima, Jalisco, Guerrero, Oaxaca y Chiapas.

Palabras clave: Phaeophyceae, inventario, estacionalidad, riqueza específica

\section{INTRODUCCIÓN}

Los primeros registros de algas pardas de la costa de Michoacán corresponden a 3 especies de Padina Adanson, registradas para Las Peñas y Caleta de Campos, en el trabajo de Chávez-Barrera (1980). Fue hasta 1990, cuando Dreckmann et al. (1990) presentaron una lista florística de las algas marinas bentónicas de la costa norte de Michoacán, donde se registraron 68 especies, 12 de las cuales pertenecen a Phaeophyceae. Posteriormente, Stout \& Dreckmann (1993), identificaron 68 especies de macroalgas en el faro de Bucerias: 15 de las cuales eran Phaeophyceae. León-Tejera et al. (1993) ubicaron 23
Phaeophyceae, 9 de las cuales fueron registros para Caleta de Campos. Por otro lado, en los estudios de MendozaGonzález et al. (2000) de la familia Sphacelariaceae en México y el de Ávila-Ortiz \& Pedroche (2005), del género Padina en la región tropical del Pacífico de México, se registran varias especies en algunas localidades del litoral de Michoacán. Finalmente, en el catálogo de Pedroche et al. (2008), se enlistan 24 especies de Phaeophyceae en la costa de Michoacán. Hasta el momento, no existe para el litoral de Michoacán un estudio enfocado exclusivamente en las algas pardas. 
Las algas pardas (Phaeophyceae) representan un componente importante de las zonas rocosas litorales y sublitorales en los ecosistemas templados y tropicales (León-Álvarez \& González-González 1993, La Barre et al. 2010). Son productores primarios, y proveen refugio y alimento a numerosos invertebrados (Mendoza-González et al. 2018). En los trabajos mencionados anteriormente se registran 25 taxones de Phaeophyceae para el litoral de Michoacán (Pedroche et al. 2008). Por tanto, el objetivo de este trabajo fue contribuir y actualizar el conocimiento taxonómico de las algas pardas que habitan la costa de Michoacán, México con el fin de reportar y describir algunos nuevos registros de Phaeophyceae para la zona de litoral de Michoacán, así como proporcionar datos de su distribución, hábitat y reproducción durante las temporadas climáticas de lluvias y secas, además de observar y describir la variación estacional de la riqueza específica y la fenología reproductiva de las Phaeophyceae.

\section{MATERIALES Y MÉTODOS}

\section{Área de ESTUdio}

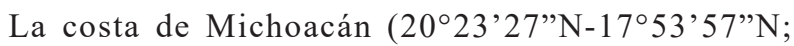
$\left.100^{\circ} 03^{\prime} 32^{\prime \prime} \mathrm{O}-103^{\circ} 44^{\prime} 49^{\prime} \mathrm{O}\right)$, es una angosta franja entre el océano Pacífico y la Sierra Madre del Sur, que se extiende de sureste a noroeste entre la Boca de San Francisco en la Barra de Zacatula y la Boca de Apiza, en la desembocadura del río Coahuayana (límite del estado de Colima). La costa michoacana termina a la mitad del canal de la Barra de San Francisco, boca del río Balsas en los límites del Michoacán y Guerrero (Brand 2013) y está formada por varios acantilados marinos de rocas metamórficas e ígneas, bahías, puntas y escotaduras. Las planicies costeras son en su mayoría estrechas, excepto aquellas que se localizan enseguida de la desembocadura del río Balsas y las que forman el valle de Coahuayana. El litoral michoacano tiene una gran cantidad de ríos y arroyos, así como lagunas y esteros (Brand 2013). El clima es del tipo Tropical subhúmedo con lluvias en verano, las cuales se extienden hasta octubre (García 1983), la temperatura anual es de $26^{\circ} \mathrm{C}$. La cubierta vegetal está constituida por bosque tropical subcaducifolio, bosque tropical caducifolio, manglar, dunas costeras y palmar (Brand 2013). La estación de lluvias se presenta de junio a octubre y la de secas de noviembre a mayo. El régimen de mareas es micromareal mixto (Mendoza-González et al. 2018). El material ficológico se obtuvo de 15 muestreos; 4 de ellos en la temporada climática de lluvias [mayo (2006, 2008, 2014); agosto (2013); septiembre (2009)] y 11 en la de secas [marzo de (2007, 2008, 2010); abril (2009); noviembre (2010); diciembre (2006, 2008, 2009, 2012)], en 11 localidades del litoral de Michoacán. En la Tabla 1 se detallan los 11 sitios de muestreo con sus coordenadas geográficas (GPS), fisiografía, exposición al oleaje, sustrato, profundidad y fecha de recolección y en la Figura 1 se muestra la distribución de las localidades en la costa de Michoacán, México.

Tabla 1. Caracterización de las localidades de muestreo de algas pardas en el litoral de Michoacán, México / Characterization of sampling sites of brown algae on the coast of Michoacán, México

\begin{tabular}{|c|c|c|c|c|c|c|}
\hline Localidades & $\begin{array}{l}\text { Coordenadas } \\
\text { GPS }\end{array}$ & Fisiografía & $\begin{array}{l}\text { Exposición } \\
\text { al oleaje }\end{array}$ & Sustrato & Profundidad & Fecha de recolecta \\
\hline San Telmo & $\begin{array}{l}18^{\circ} 37^{\prime} 29^{\prime \prime} \mathrm{N} \\
103^{\circ} 41^{\prime} 06^{\prime \prime} \mathrm{O}\end{array}$ & $\begin{array}{l}\text { Arenosa con } \\
\text { acantilados, agregados } \\
\text { rocosos }\end{array}$ & Expuesto & Rocoso & $0-1 \mathrm{~m}$ & $12 / 2009$ \\
\hline San Juan de Alima & $\begin{array}{l}18^{\circ} 34^{\prime} 59^{\prime \prime} \mathrm{N} \\
103^{\circ} 40^{\prime} 28^{\prime \prime} \mathrm{O}\end{array}$ & $\begin{array}{l}\text { Arenosa con agregados } \\
\text { rocosos }\end{array}$ & Expuesto & Rocoso & $0-1 \mathrm{~m}$ & $12 / 2012,08 / 2013$ \\
\hline $\begin{array}{l}\text { El Zapote de } \\
\text { Madero-La Mesa }\end{array}$ & $\begin{array}{l}18^{\circ} 23^{\prime} 10^{\prime \prime} \mathrm{N} \\
103^{\circ} 31^{\prime} 35^{\prime \prime} \mathrm{O}\end{array}$ & $\begin{array}{l}\text { Arenosa con plataformas } \\
\text { rocosas y agregados de } \\
\text { rocas }\end{array}$ & $\begin{array}{l}\text { Expuesto y } \\
\text { protegido }\end{array}$ & $\begin{array}{l}\text { Rocoso con pozas } \\
\text { intermareales }\end{array}$ & $0-1 \mathrm{~m}$ & $\begin{array}{l}03,05 / 2006,03, \\
06 / 2007,09,12 / 2008, \\
12 / 2009,03 / 2010,08, \\
12 / 2012,08 / 2013\end{array}$ \\
\hline La Majahuita & $\begin{array}{l}18^{\circ} 2^{\prime} 413^{\prime \prime} \mathrm{N} \\
103^{\circ} 31^{\prime} 39^{\prime \prime} \mathrm{O}\end{array}$ & $\begin{array}{l}\text { Rocosa con plataforma } \\
\text { de marea }\end{array}$ & $\begin{array}{l}\text { Expuesto y } \\
\text { protegido }\end{array}$ & $\begin{array}{l}\text { Rocoso con pozas } \\
\text { intermareales con } \\
\text { canales de corriente }\end{array}$ & $0-1 \mathrm{~m}$ & $\begin{array}{l}01,03,09 / 2008 \\
04 / 2009,03 / 2010 \\
12 / 2012\end{array}$ \\
\hline Maruata-El Faro & $\begin{array}{l}18^{\circ} 16^{\prime} 18^{\prime \prime} \mathrm{N} \\
103^{\circ} 21^{\prime} 20^{\prime \prime} \mathrm{O}\end{array}$ & $\begin{array}{l}\text { Arenosa con } \\
\text { acantilados, agregados } \\
\text { rocosos }\end{array}$ & Expuesto & Rocoso & $0-2 \mathrm{~m}$ & $08 / 2013,12 / 2012$ \\
\hline Carrizalillo & $\begin{array}{l}18^{\circ} 03^{\prime} 33^{\prime \prime} \mathrm{N} \\
102.42^{\prime} 11^{\prime \prime} \mathrm{O}\end{array}$ & $\begin{array}{l}\text { Arenosa con agregados } \\
\text { rocosos }\end{array}$ & Expuesto & Rocoso con pozas & $0-1 \mathrm{~m}$ & $01,04 / 2009,03 / 2010$ \\
\hline Caleta de Campos & $\begin{array}{l}18^{\circ} 04^{\prime} 26^{\prime \prime} \mathrm{N} \\
102^{\circ} 45^{\prime} 23^{\prime \prime} \mathrm{O}\end{array}$ & $\begin{array}{l}\text { Arenosa con acantilados, } \\
\text { agregados rocosos }\end{array}$ & Expuesto & Rocoso, acantilados & $0-1 \mathrm{~m}$ & $04 / 2009,12 / 2012$ \\
\hline Caletilla & $\begin{array}{l}18^{\circ} 03^{\prime} 01^{\prime \prime} \mathrm{N} \\
102^{\circ} 38^{\prime} 58^{\prime \prime} \mathrm{O}\end{array}$ & $\begin{array}{l}\text { Arenosa con } \\
\text { plataformas rocosas y } \\
\text { agregados de rocas }\end{array}$ & $\begin{array}{l}\text { Expuesto y } \\
\text { protegido }\end{array}$ & $\begin{array}{l}\text { Rocoso con pozas } \\
\text { intermareales }\end{array}$ & $0-1 \mathrm{~m}$ & $\begin{array}{l}12 / 2006,03 / 2007 \\
03 / 2008,04 / 2009 \\
03 / 2010\end{array}$ \\
\hline La Saladita & $\begin{array}{l}18^{\circ} 03^{\prime} 06^{\prime \prime} \mathrm{N} \\
102^{\circ} 37^{\prime} 35^{\prime \prime} \mathrm{O}\end{array}$ & $\begin{array}{l}\text { Arenosa con agregados } \\
\text { rocosos }\end{array}$ & Expuesto & $\begin{array}{l}\text { Rocoso con pozas } \\
\text { intermareales }\end{array}$ & $0-1 \mathrm{~m}$ & $\begin{array}{l}03 / 2007,12 / 2008 \\
04 / 2009\end{array}$ \\
\hline $\begin{array}{l}\text { Tres Palmas- } \\
\text { Playa Rangel }\end{array}$ & $\begin{array}{l}18^{\circ} 01^{\prime} 14^{\prime \prime} \mathrm{N} \\
102^{\circ} 30^{\prime} 06^{\prime \prime} \mathrm{O}\end{array}$ & $\begin{array}{l}\text { Arenosa con agregados } \\
\text { rocosos }\end{array}$ & Expuesto & $\begin{array}{l}\text { Rocoso con pozas } \\
\text { intermareales }\end{array}$ & $0-1 \mathrm{~m}$ & $\begin{array}{l}05 / 2008,08 / 2013 \\
05 / 2014\end{array}$ \\
\hline Las Peñas & $\begin{array}{l}18^{\circ} 01^{\prime} 14^{\prime \prime} \mathrm{N} \\
102^{\circ} 30^{\prime} 6^{\prime \prime} \mathrm{O}\end{array}$ & $\begin{array}{l}\text { Arenosa con } \\
\text { acantilados, agregados } \\
\text { rocosos }\end{array}$ & Expuesto & $\begin{array}{l}\text { Rocoso con pozas } \\
\text { intermareales }\end{array}$ & $0-1 \mathrm{~m}$ & $\begin{array}{l}12 / 2008,04,12 / 2009, \\
03 / 2010\end{array}$ \\
\hline
\end{tabular}




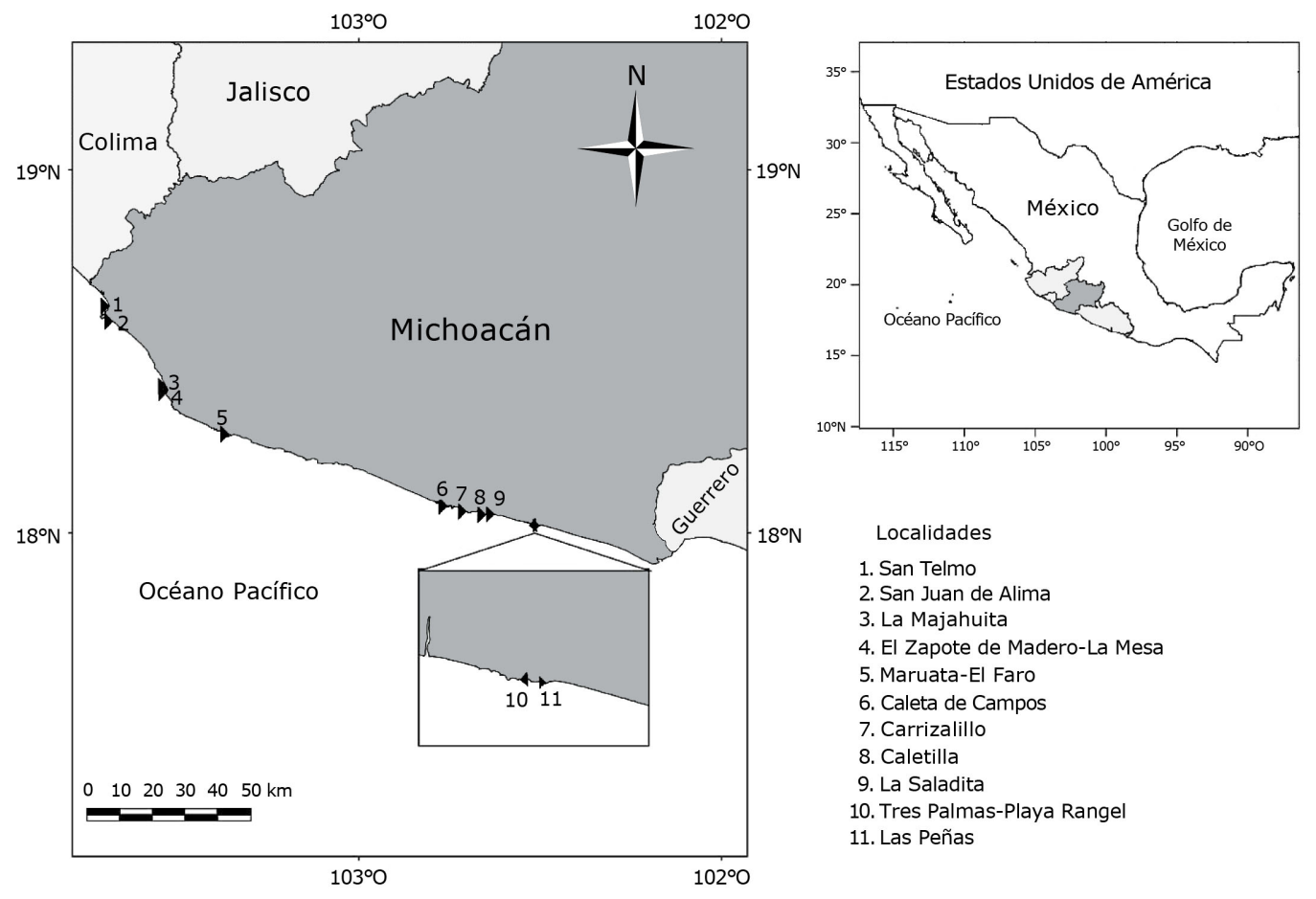

Figura 1. Mapa del área de estudio y las localidades de muestreo (1-11) en el litoral de Michoacán, México / Map of the study area and sampling locaties (1-11) on the coast of Michoacán, Mexico

\section{RECOLECCIÓN DE MATERIAL FICOLÓGICO}

Las algas se recolectaron manualmente en el nivel intermareal con espátulas, y se fijaron en formaldehído al $5 \%$ en agua de mar. Las recolectas se realizaron en segmentos de playa de alrededor de 500-1.500 m de extensión. El material fue determinado, herborizado y depositado en el herbario de la Escuela Nacional de Ciencias Biológicas-ENCB (Thiers 2017). Se hicieron cortes de los talos con navajas de doble filo para diferenciar estructuras reproductivas y vegetativas. Para la observación de las algas se utilizó un microscopio binocular Olympus ${ }^{\circledR}$ CX31RBSF, con cámara digital integrada. La determinación de las Phaeophyceae se hizo usando los trabajos de Setchell \& Gardner (1924), Taylor (1945, 1960), Fletcher (1987), Schneider \& Searles (1991) y Norris (2010).

La secuencia taxonómica de la lista florística sigue el orden propuesto por Wynne (2017). La actualización nomenclatural se basó en Wynne (2017) y Guiry \& Guiry (2019). Cada especie se acompañó con datos sobre distribución en el área de estudio, estacionalidad, estado reproductivo, hábitat, epifitismo y número de herbario o de recolección.

\section{Resultados}

Las localidades de estudio establecidas en el litoral de Michoacán tienen sustrato predominantemente rocoso y 31 de las 41 especies de algas pardas ubicadas en este estudio habitaban en plataformas rocosas, pozas de marea, sobre guijarros, cantos rodados, zonas de acantilados y canales de corriente. Mientras que 12 de ellas son epífitas, y de estas, 6 se ubicaron tanto en sustrato rocoso como epífitas (Tabla 2). La mayor parte de las especies ubicadas en este estudio tienen distribución en mares tropicales del océano Pacífico, Atlántico e Índico. Como se indica en la Tabla 2, la mayoría de las especies se ubicó en aquellas localidades con sustrato rocoso como plataformas, pozas y canales de corriente. 
Tabla 2. Algas pardas (Phaeophyceae) del litoral de Michoacán, México. La explicación de los símbolos está al final de la tabla / Brown algae (Phaeophyceae) from the coast of Michoacán. The explanation of the symbols is at the end of the table

\begin{tabular}{|c|c|c|c|c|c|c|c|}
\hline Especies & Localidades & Est. & Reproducción & Habitat & OBS. & Referencias & $\begin{array}{c}\text { Número } \\
\text { Herbario } \\
\text { ENCB }\end{array}$ \\
\hline \multicolumn{8}{|l|}{ OCHROPHYTA } \\
\hline \multicolumn{8}{|l|}{ Phaeophyceae } \\
\hline \multicolumn{8}{|l|}{ Dictyotales } \\
\hline \multicolumn{8}{|l|}{ Dictyotaceae } \\
\hline 1. Dictyopteris delicatula J.V.Lamouroux & 7 & $\mathrm{~S}$ & $\mathrm{Ve}$ & R, Epi & NRM & A & 25097 \\
\hline 2. Dictyota bartayresiana & 3,4 & $\mathrm{Ll}$ & Esp & R, Epi & & $\mathrm{A}, \mathrm{C}, \mathrm{D}, \mathrm{G}$ & 21159 \\
\hline \multirow{2}{*}{ J.V.Lamouroux } & & $\mathrm{S}$ & & & & & 24072 \\
\hline & & & & & & & 24436 \\
\hline 3. D. concrescens W.R.Taylor & 2 & $\mathrm{~S}$ & Esp & $\mathrm{R}$ & & $A, E, G$ & 25098 \\
\hline \multirow{7}{*}{ 4. D. crenulata J.Agardh } & $3,4,8,10$ & $\mathrm{~L} 1$ & Esp Oo An & $\mathrm{R}, \mathrm{Pm}$ & NRM & A & 21146 \\
\hline & 11 & $\mathrm{~S}$ & & & & & 21155 \\
\hline & & & & & & & 21156 \\
\hline & & & & & & & 21180 \\
\hline & & & & & & & 21186 \\
\hline & & & & & & & 22626 \\
\hline & & & & & & & 24073 \\
\hline $\begin{array}{l}\text { 5.D. dichotoma (Hudson) } \\
\text { J.V.Lamouroux }\end{array}$ & & & & & & E, G & \\
\hline 6. D. dichotoma var. intricata & 7,8 & $\mathrm{~S}$ & $\mathrm{Ve}$ & $\mathrm{R}, \mathrm{Pm}$ & NRM & A & 19622 \\
\hline (C.Agardh) Greville & & & & & & & 25096 \\
\hline 7. D. friabilis Setchell & 4,8 & $\mathrm{~S}$ & $\mathrm{Ve}$ & $\mathrm{R}, \mathrm{Pm}$ & NRM & A & $\begin{array}{l}21140 \\
22629\end{array}$ \\
\hline $\begin{array}{l}\text { 8. D. implexa (Desfontaines) } \\
\text { J.V.Lamouroux }\end{array}$ & & & & & & $\mathrm{E}, \mathrm{G}$ & \\
\hline $\begin{array}{l}\text { 9. D. pinnata (E.Y. Dawson) I.Hörnig, } \\
\text { R.Schnetter \& Prud'homme van Reine }\end{array}$ & & & & & & E, G & \\
\hline 10.D. vivesii M.Howe & 10 & $\mathrm{~S}$ & $\mathrm{Ve}$ & $\mathrm{R}$ & & A, D & 21128 \\
\hline $\begin{array}{l}\text { 11. Lobophora variegata (J.V. } \\
\text { Lamouroux) Womersley ex } \\
\text { E.C.Oliveira }\end{array}$ & 8 & $\mathrm{~S}$ & $\mathrm{Ve}$ & $\mathrm{R}$ & & A, D, G & 25100 \\
\hline 12. Padina concrescens Thivy & & & & & & $\mathrm{D}, \mathrm{G}$ & \\
\hline \multirow[t]{8}{*}{ 13. P. crispata Thivy } & $1,2,3,4,5$, & $\mathrm{Ll}$ & Esp Og & $\mathrm{R}$ & & $\mathrm{A}, \mathrm{B}, \mathrm{C}, \mathrm{G}$ & 21135 \\
\hline & $6,8,9,10$ & $\mathrm{~S}$ & & & & & 21143 \\
\hline & 11 & & & & & & 21148 \\
\hline & & & & & & & 21149 \\
\hline & & & & & & & 21185 \\
\hline & & & & & & & 21186 \\
\hline & & & & & & & 21187 \\
\hline & & & & & & & 22627 \\
\hline \multirow[t]{9}{*}{ 14. P. durvillei Bory } & $2,3,4,6,7$ & $\mathrm{Ll}$ & Esp Og & $\mathrm{R}, \mathrm{Pm}$ & & $\mathrm{A}, \mathrm{B}, \mathrm{C}, \mathrm{G}$ & 21144 \\
\hline & $8,9,10$ & $\mathrm{~S}$ & & & & & 21145 \\
\hline & & & & & & & 21160 \\
\hline & & & & & & & 21172 \\
\hline & & & & & & & 21189 \\
\hline & & & & & & & 21190 \\
\hline & & & & & & & 24074 \\
\hline & & & & & & & 24075 \\
\hline & & & & & & & 24626 \\
\hline 15. P. mexicana E.Y.Dawson & 4 & $\mathrm{~L} 1$ & Esp & $\mathrm{R}$ & NRM & A & 24082 \\
\hline \multirow[t]{6}{*}{ 16. P. mexicana var. erecta Ávila-Ortiz } & $3,4,7$ & $\mathrm{~S}$ & Esp Og & $\mathrm{R}$ & NRM & A & 21136 \\
\hline & & & & & & & 21164 \\
\hline & & & & & & & 21165 \\
\hline & & & & & & & 21174 \\
\hline & & & & & & & 24083 \\
\hline & & & & & & & 24084 \\
\hline $\begin{array}{l}\text { 17. P. ramonribae Ávila-Ortiz, Pedroche } \\
\text { \& Díaz-Martínez }\end{array}$ & 3 & $\mathrm{~S}$ & Esp & $\mathrm{R}$ & NRM & A & 24085 \\
\hline $\begin{array}{l}\text { 18. Spatoglossum lanceolatum } \\
\text { E.Y.Dawson }\end{array}$ & 4 & $\mathrm{~S}$ & $\mathrm{Og}$ & $\mathrm{R}$ & NRM & A & 21153 \\
\hline 19. S. subflabellatum E.Y.Dawson & 3,4 & $\mathrm{~S}$ & Esp & $\mathrm{R}$ & NRM & A & 24086 \\
\hline $\begin{array}{l}\text { 20. Zonaria farlowii Setchell \& } \\
\text { N.L.Gardner }\end{array}$ & & & & & & E & \\
\hline
\end{tabular}

Localidades: 1. San Telmo, 2. San Juan de Alima, 3. La Majahuita, 4. El Zapote de Madero-La Mesa, 5. Maruata-El Faro, 6. Caleta de Campos, 7. Carrizalillo, 8. Caletilla, 9. La Saladita, 10. Tres Palmas-Playa Rangel, 11. Las Peñas. Est. (Estacionalidad): Ll= Lluvias (Mayo de 2006, 2008, 2014; Agosto 2013; Septiembre 2009), S= Secas (Marzo de 2007, 2008, 2010; Abril 2009; Noviembre 2010; Diciembre de 2006, 2008, 2009; 2012). Reproducción: Og= Oogonios; An= Anteridios; Esp= Esporangios; Ug= Unangios; $\mathrm{Plu}=$ Plurangios: $\mathrm{Pp}=$ Propágulos; $\mathrm{Ve}=$ Vegetativo. Hábitat: $\mathrm{R}=$ Rocoso; $\mathrm{Pm}=$ Pozas intermareales; $\mathrm{Epi}=$ Epífito. Obs. (Observaciones): $\mathrm{NRM}=$ Nuevo registro para Michoacán; $\mathrm{NRP}=$ Nuevo registro para la costa del Pacífico de México. Referencias: A) Este estudio, B) Chávez-Barrera (1980); C) Dreckmann et al. (1990); D) León-Tejera et al. (1993); E) Stout \& Dreckmann (1993); F) León-Álvarez \& González-González (1993); G) Pedroche et al. (2008) 


\begin{tabular}{|c|c|c|c|c|c|c|c|}
\hline Especies & Localidades & Est. & Reproducción & Habitat & OBS. & Referencias & $\begin{array}{l}\text { Número } \\
\text { Herbario } \\
\text { ENCB }\end{array}$ \\
\hline \multicolumn{8}{|l|}{$\begin{array}{l}\text { Ectocarpales } \\
\text { Acinetosporaceae }\end{array}$} \\
\hline $\begin{array}{l}\text { 21. Feldmannia indica (Sonder) } \\
\text { Womersley \& A.Bailey }\end{array}$ & & & & & & $\mathrm{C}, \mathrm{E}, \mathrm{G}$ & \\
\hline 22. F. irregularis (Kützing) Hamel & $3,5,8$ & $\begin{array}{l}\mathrm{Ll} \\
\mathrm{S}\end{array}$ & Plu & R, Epi & NRM & A & $\begin{array}{l}21161 \\
24004 \\
24128 \\
24129\end{array}$ \\
\hline 23. F. mitchelliae (Harvey) H.-S.Kim & $6,7,9$ & $\begin{array}{l}\mathrm{L} 1 \\
\mathrm{~S}\end{array}$ & Plu & R, Epi & & $\mathrm{A}, \mathrm{E}, \mathrm{G}$ & $\begin{array}{l}21129 \\
21168 \\
23842\end{array}$ \\
\hline $\begin{array}{l}\text { 24. F. simplex (P. Crouan \& H.Crouan) } \\
\text { Hamel }\end{array}$ & 3 & $\mathrm{~S}$ & Plu & Epi & NRM & A & $\begin{array}{l}21135 \\
21148\end{array}$ \\
\hline $\begin{array}{l}\text { 25. Hincksia sandriana (Zanardini) } \\
\text { P.C.Silva }\end{array}$ & & & & & & C, E, G & \\
\hline $\begin{array}{l}\text { 26. H. saundersii (Setchell \& } \\
\text { N.L.Gardner) P.C.Silva } \\
\text { Chordariaceae }\end{array}$ & & & & & & $\mathrm{D}, \mathrm{E}, \mathrm{G}$ & \\
\hline $\begin{array}{l}\text { 27. Compsonema intricatum Setchell \& } \\
\text { N.L.Gardner }\end{array}$ & 3,4 & $\mathrm{~S}$ & Plu & Epi & NRM & A & $\begin{array}{l}21121 \\
21121\end{array}$ \\
\hline 28. C. serpens Setchell \& N.L.Gardner & $3,4,6$ & $\begin{array}{l}\mathrm{Ll} \\
\mathrm{S}\end{array}$ & Plu & Epi & NRM & A & $\begin{array}{l}21177 \\
21169 \\
24435\end{array}$ \\
\hline $\begin{array}{l}\text { 29. Hecatonema streblonematoides } \\
\text { (Setchell \& N.L.Gardner) Loiseaux }\end{array}$ & $3,4,5,9$ & $\begin{array}{l}\mathrm{Ll} \\
\mathrm{S}\end{array}$ & Plu & Epi & NRM & A & $\begin{array}{l}23948 \\
23953 \\
24031 \\
24031 \\
24089\end{array}$ \\
\hline $\begin{array}{l}\text { 30. Kuetzingiella elachistaeformis } \\
\text { (Heydrich) M.Balakrishnan \& Kinkar }\end{array}$ & 3 & $\begin{array}{l}\mathrm{Ll} \\
\mathrm{S}\end{array}$ & Plu & Epi & NRM & A & 24079 \\
\hline $\begin{array}{l}\text { 31. Streblonema anomalum Setchell \& } \\
\text { N.L.Gardner } \\
\text { Ectocarpaceae }\end{array}$ & 5 & $\mathrm{Ll}$ & Plu & Epi & NRP & A & \\
\hline $\begin{array}{l}\text { 32. Ectocarpus commensalis Setchell \& } \\
\text { N.L.Gardner }\end{array}$ & 9,11 & $\mathrm{~S}$ & Plu & Epi & NRM & A & $\begin{array}{l}21124 \\
21142\end{array}$ \\
\hline $\begin{array}{l}\text { 33. E. gonodioides Setchell \& } \\
\text { N.L.Gardner }\end{array}$ & 3,4 & $\mathrm{~S}$ & Plu & Epi & NRM & A & $\begin{array}{l}23877 \\
24060 \\
24061\end{array}$ \\
\hline 34. E. siliculosus (Dillwyn) Lyngbye & $\begin{array}{l}3,5,6,9 \\
11\end{array}$ & $\begin{array}{l}\mathrm{Ll}, \\
\mathrm{S}\end{array}$ & Plu & R, Epi & NRM & A & $\begin{array}{l}23884 \\
23924 \\
23925 \\
24087 \\
24626\end{array}$ \\
\hline $\begin{array}{l}\text { 35. E. siliculosus var. Dasycarpus } \\
\text { (Kuckuck) Gallardo }\end{array}$ & 3,4 & $\mathrm{~S}$ & Plu & $\mathrm{R}$ & & $\mathrm{A}, \mathrm{D}, \mathrm{G}$ & $\begin{array}{l}24085 \\
24088\end{array}$ \\
\hline $\begin{array}{l}\text { 36. E. taoniae Setchell \& N.L.Gardner } \\
\text { Scytosiphonaceae }\end{array}$ & 2,3 & $\mathrm{~S}$ & Plu & Epi & NRP & A & 21187 \\
\hline $\begin{array}{l}\text { 37. Chnoospora minima (Hering) } \\
\text { Papenfuss }\end{array}$ & $\begin{array}{l}1,2,3,4,6 \\
7,8,9,11\end{array}$ & $\begin{array}{l}\mathrm{L} 1 \\
\mathrm{~S}\end{array}$ & $\mathrm{Ve}$ & $\mathrm{R}$ & & $\mathrm{A}, \mathrm{C}, \mathrm{E}, \mathrm{G}$ & $\begin{array}{l}21170 \\
21171 \\
24132 \\
24133 \\
24134 \\
24135 \\
24136 \\
24137 \\
24140 \\
24141\end{array}$ \\
\hline $\begin{array}{l}\text { 38. Colpomenia sinuosa (Mertens ex } \\
\text { Roth) Derbès \& Solier }\end{array}$ & 3,4 & $\mathrm{~S}$ & $\mathrm{Ug}$ & $\mathrm{R}$ & NRM & A & 21141 \\
\hline $\begin{array}{l}\text { 39. Hapterophycus anastomosans } \\
\text { E.Y.Dawson }\end{array}$ & 3 & $\mathrm{~S}$ & $\mathrm{Ve}$ & $\mathrm{R}$ & NRM & A & 21156 \\
\hline $\begin{array}{l}\text { 40. Rosenvingea intricata (J.Agardh) } \\
\text { Børgesen }\end{array}$ & 10 & $\mathrm{~S}$ & Ug & $\mathrm{R}$ & NRM & A & 21173 \\
\hline
\end{tabular}

Localidades: 1. San Telmo, 2. San Juan de Alima, 3. La Majahuita, 4. El Zapote de Madero-La Mesa, 5. Maruata-El Faro, 6. Caleta de Campos, 7. Carrizalillo, 8. Caletilla, 9. La Saladita, 10. Tres Palmas-Playa Rangel, 11. Las Peñas. Est. (Estacionalidad): $\mathrm{Ll}=$ Lluvias (Mayo de 2006, 2008, 2014; Agosto 2013; Septiembre 2009), S= Secas (Marzo de 2007, 2008, 2010; Abril 2009; Noviembre 2010; Diciembre de 2006, 2008, 2009; 2012). Reproducción: Og= Oogonios; An= Anteridios; Esp= Esporangios; Ug= Unangios; $\mathrm{Plu}=$ Plurangios: $\mathrm{Pp}=$ Propágulos; Ve= Vegetativo. Hábitat: $\mathrm{R}=$ Rocoso; $\mathrm{Pm}=$ Pozas intermareales; Epi $=$ Epífito. Obs. $($ Observaciones): $\mathrm{NRM}=$ Nuevo registro para Michoacán; $\mathrm{NRP}=\mathrm{Nuevo}$ registro para la costa del Pacífico de México. Referencias: A) Este estudio, B) Chávez-Barrera (1980); C) Dreckmann et al. (1990); D) León-Tejera et al. (1993); E) Stout \& Dreckmann (1993); F) León-Álvarez \& González-González (1993); G) Pedroche et al. (2008) 
Tabla 2. Continuación / Continued

\begin{tabular}{|c|c|c|c|c|c|c|c|}
\hline Especies & Localidades & Est. & Reproducción & Habitat & OBS. & Referencias & $\begin{array}{c}\text { Número } \\
\text { Herbario } \\
\text { ENCB }\end{array}$ \\
\hline \multicolumn{8}{|l|}{ Scytothamnales } \\
\hline \multicolumn{8}{|l|}{ Asteronemataceae } \\
\hline 41. Asteronema breviarticulatum & 2,3 & $\mathrm{Ll}$ & Plu & $\mathrm{R}$ & & $\mathrm{D}, \mathrm{E}, \mathrm{G}$ & 21179 \\
\hline \multicolumn{8}{|l|}{ Bachelotiaceae } \\
\hline \multirow{3}{*}{$\begin{array}{l}\text { 42. Bachelotia antillarum (Grunow) } \\
\text { Gerloff }\end{array}$} & $3,4,5$ & $\mathrm{Ll}$ & Plu & R, Epi & NRM & A & 24006 \\
\hline & & $\mathrm{s}$ & & & & & 24007 \\
\hline & & & & & & & 24008 \\
\hline \multicolumn{8}{|l|}{ Sphacelariales } \\
\hline \multicolumn{8}{|l|}{ Lithodermataceae } \\
\hline \multirow{2}{*}{\multicolumn{8}{|c|}{$\begin{array}{l}\text { 43. Pseudolithoderma nigrum Hollenberg } \\
\text { Sphacelariaceae }\end{array}$}} \\
\hline & & & & & & & \\
\hline 44. Sphacelaria novae-hollandiae Sonder & $3,4,10$ & $\mathrm{Ll}$ & $\mathrm{Pp}$ & $\mathrm{R}$ & NRM & A & 21130 \\
\hline \multirow{3}{*}{ 45. S. rigidula Kützing } & $2,3,4$ & L1 & $\mathrm{Pp}$ & Epi & & A, G & $\begin{array}{l}24144 \\
21182\end{array}$ \\
\hline & & $\mathrm{S}$ & & & & & 21184 \\
\hline & & & & & & & 24628 \\
\hline 46. S. tribuloides Meneghini & 9 & $\mathrm{~s}$ & $\mathrm{Pp}$ & $\mathrm{R}$ & NRM & A & 25101 \\
\hline & & & & & & & \\
\hline \multicolumn{8}{|l|}{ Hapalospongidiaceae } \\
\hline \multicolumn{8}{|l|}{ 47. Hapalospongidion gelatinosum De } \\
\hline \multirow{2}{*}{\multicolumn{7}{|c|}{$\begin{array}{l}\text { A.Saunders } \\
\text { Neoralfsiaceae }\end{array}$}} & \\
\hline & & & & & & & \\
\hline 48. Neoralfsia hancockii (E.Y.Dawson) & $3,4,5,6$ & $\mathrm{~s}$ & Ug & $\mathrm{R}$ & & $\mathrm{A}, \mathrm{C}, \mathrm{F}, \mathrm{G}$ & 21126 \\
\hline \multirow{3}{*}{$\begin{array}{l}\text { D.León-Álvarez \& M.L.Núñez- } \\
\text { Reséndiz }\end{array}$} & & & & & & & 23936 \\
\hline & & & & & & & 23937 \\
\hline & & & & & & & 24707 \\
\hline \multicolumn{8}{|l|}{ Ralfsiaceae } \\
\hline 49. Ralfsia confusa Hollenberg & 8 & $\mathrm{~S}$ & $\mathrm{Ug}$ & $\mathrm{R}$ & & $\mathrm{A}, \mathrm{C}, \mathrm{F}, \mathrm{G}$ & 18783 \\
\hline \multirow{2}{*}{\multicolumn{8}{|c|}{ Fucales }} \\
\hline & & & Sargassaceae & & & & \\
\hline \multirow{7}{*}{ 51. Sargassum howellii Setchell } & $3,4,8$ & $\mathrm{Ll}$ & Og An & $\mathrm{R}$ & NRM & A & 21123 \\
\hline & & $\mathrm{s}$ & & & & & 21178 \\
\hline & & & & & & & 22632 \\
\hline & & & & & & & 24126 \\
\hline & & & & & & & 24127 \\
\hline & & & & & & & 24128 \\
\hline & & & & & & & 24129 \\
\hline \multirow[t]{5}{*}{ 52. S. liebmannii J.Agardh } & $3,4,5,8$ & $\mathrm{~L} 1$ & Og An & $\mathrm{R}$ & & $\mathrm{A}, \mathrm{C}, \mathrm{D}, \mathrm{G}$ & \\
\hline & & $\mathrm{s}$ & & & & & 21120 \\
\hline & & & & & & & 21121 \\
\hline & & & & & & & 21177 \\
\hline & & & & & & & 24130 \\
\hline
\end{tabular}

Localidades: 1. San Telmo, 2. San Juan de Alima, 3. La Majahuita, 4. El Zapote de Madero-La Mesa, 5. Maruata-El Faro, 6. Caleta de Campos, 7. Carrizalillo, 8. Caletilla, 9. La Saladita, 10. Tres Palmas-Playa Rangel, 11. Las Peñas. Est. (Estacionalidad): Ll= Lluvias (Mayo de 2006, 2008, 2014; Agosto 2013; Septiembre 2009), S= Secas (Marzo de 2007, 2008, 2010; Abril 2009; Noviembre 2010; Diciembre de 2006, 2008, 2009; 2012). Reproducción: Og= Oogonios; An= Anteridios; $\mathrm{Esp}=$ Esporangios; $\mathrm{Ug}=$ Unangios; $\mathrm{Plu}=$ Plurangios: $\mathrm{Pp}=$ Propágulos; $\mathrm{Ve}=$ Vegetativo. Hábitat: $\mathrm{R}=\mathrm{Rocoso}$; $\mathrm{Pm}=$ Pozas intermareales; Epi $=$ Epífito. Obs. (Observaciones): $\mathrm{NRM}=$ Nuevo registro para Michoacán; $\mathrm{NRP}=\mathrm{Nuevo}$ registro para la costa del Pacífico de México. Referencias: A) Este estudio, B) Chávez-Barrera (1980); C) Dreckmann et al. (1990); D) León-Tejera et al. (1993); E) Stout \& Dreckmann (1993); F) León-Álvarez \& González-González (1993); G) Pedroche et al. (2008)

\section{RIQUEZA TAXONÓMICA}

En la costa de Michoacán, un total de 41 especies de algas pardas fueron determinadas. Estas especies estuvieron distribuidas en 6 órdenes, 11 familias y 19 géneros (Tabla 2). Los géneros con mayor riqueza específica fueron Dictyota J.V.Lamouroux, Ectocarpus Lyngbye y Padina Adanson con 5 especies cada uno. Entre los géneros monoespecíficos se encontraron: Asteronema Delépine \& Asensi, Bachelotia (Bornet) Kuckuck ex Hamel, Chnoospora J.Agardh, Colpomenia (Endlicher) Derbès \& Solier, Hapterophycus Setchell \& N.L.Gardner y Neoralfsia P.-E.Lim \& H.Kawai.
Las familias mejor representadas de acuerdo con el número de taxa fueron Dictyotaceae (15 especies), siguen en importancia Ectocarpaceae y Chordariaceae ( 5 especies cada una) y Scytosiphonaceae (4 especies). Además, se ubicaron especies de Sphacelariaceae y Sargassaceae representadas por 2 o 3 especies. En la Tabla 2 se indica el detalle de las especies encontradas en este estudio. 


\section{RIQUEZA ESPECÍFICA}

Los valores de riqueza específica total oscilaron desde un máximo de 27 especies en La Majahuita, hasta un mínimo de 2 en San Telmo. El mayor número de especies se registró en localidades con plataformas y zonas rocosas expuestas en donde se forman pozas y canales de corriente como en La Majahuita (27), Zapote de Madero-La Mesa (21), Caletilla (11) y La Saladita (8). En estos sitios se ubicaron especies de las familias Dictyotaceae, Chordariaceae, Ectocarpaceae y Sphacelariaceae. Las especies con la distribución más amplia en el área de estudio fueron Padina crispata, Padina durvillei y Chnoospora minima, encontradas en 10, 8 y 9 localidades de muestreo, respectivamente. Dictyota crenulata se ubicó en 6 localidades, Ectocarpus siliculosus en 5 y finalmente Sargassum liebmannii en 4.

Del total de las especies determinadas, 38 se ubicaron en la estación climática de secas, incremento atribuido a los representantes del orden Dictyotales y Ectocarpales, en contraste con la época de lluvias con 19 especies.

\section{DESCRIPCIONES DE ALGUNOS NUEVOS REGISTROS}

Streblonema anomalum Setchell \& N.L.Gardner (Fig. 2a, b, c, d)

Talos microscópicos, parcialmente endófitos confluentes, con porciones basales que penetran en el hospedero, moderada e irregularmente ramificados, con células de 7-9 $\mu \mathrm{m}$ y 8-14 $\mu \mathrm{m}$ longitud. Filamentos erguidos de 200$300 \mu \mathrm{m}$ de alto, simples, cilíndricos, ápices redondeados, en ocasiones las células de los filamentos se dividen y entonces los filamentos se observan polisifónicos de 15-18 $\mu \mathrm{m}$ de diámetro. Células de 8-10 $\mu \mathrm{m}$ de diámetro y 7,5-15 $\mu \mathrm{m}$ de longitud, con un plasto parietal.

Reproducción: Plurangios elipsoidales u ovoides de 16-20 $\mu \mathrm{m}$ de diámetro y 40-56 $\mu \mathrm{m}$ de longitud, sésiles o pedunculados y que nacen de los filamentos reptantes.

Hábitat: Endófito de Dermonema virens (J.Agardh) Pedroche \& Ávila-Ortiz, intermareal expuesto.

Distribución: Pacífico de América (EEUU, California), México (Maruata-El Faro, Michoacán) y China (Mar Amarillo).
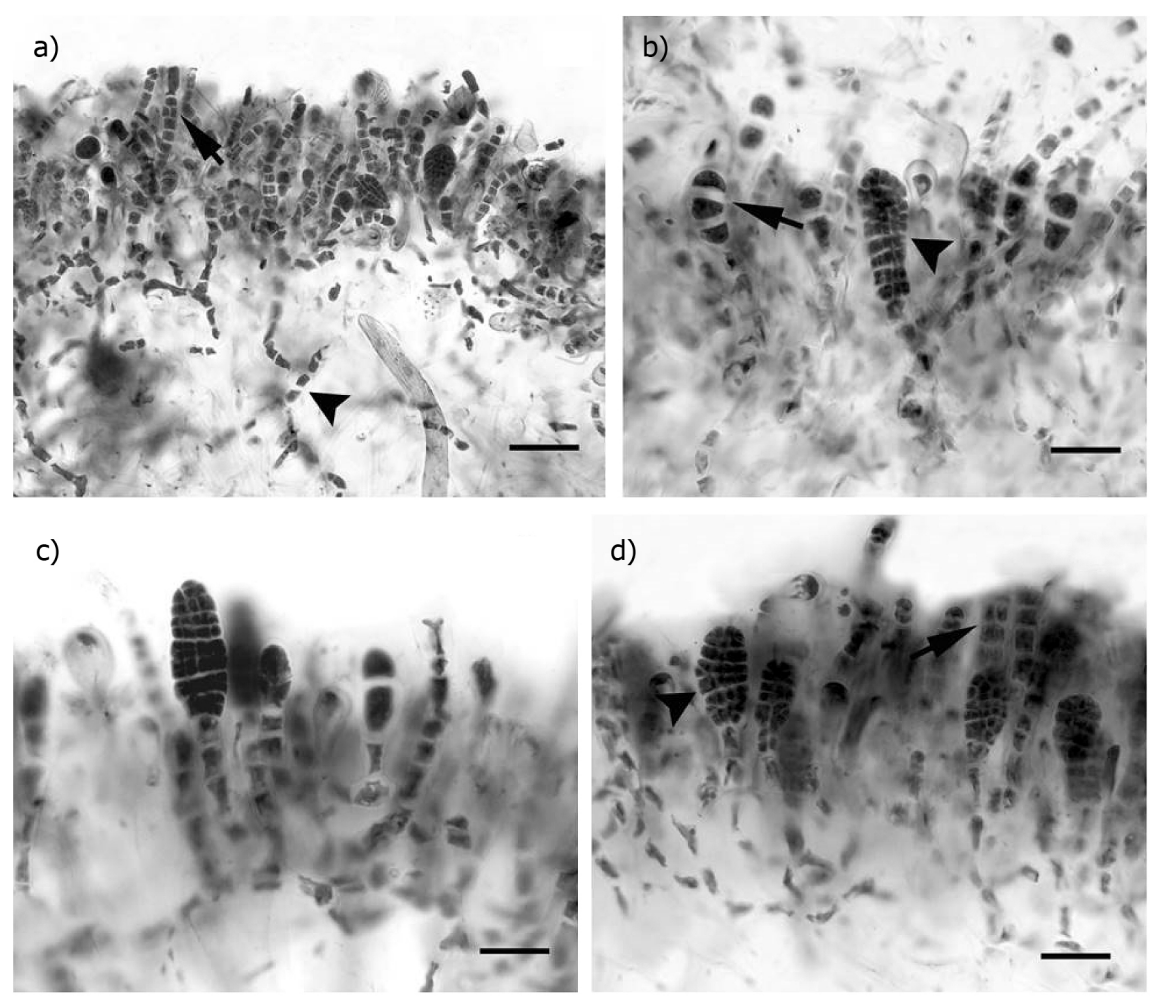

Figura 2. Streblonema anomalum. a) porción que emerge del hospedero con filamentos biseriados (flecha) y filamentos endófitos (cabeza de flecha). Escala $50 \mu \mathrm{m}, \mathrm{b}$ ) estructuras de reproducción en desarrollo (flecha) y plurangios maduros (cabeza de flecha). Escala $19 \mu \mathrm{m}$, c) estructuras de reproducción en diferentes estadios de desarrollo. Escala $27 \mu \mathrm{m}, \mathrm{d}$ ) plurangio maduro (cabeza de flecha) y filamentos biseriados (flechas) en la superficie del talo. Escala $\mathbf{3 0} \boldsymbol{\mu \mathrm { m }}$ / Streblonema anomalum. a) portion that emerges from the host with biseriate filaments (arrow) and endophytic filaments (arrowhead). Scale $50 \mu \mathrm{m}, \mathrm{b}$ ) reproductive structures in development (arrow) and mature plurilocular sporangia (arrowhead). Scale $19 \mu \mathrm{m}$, c) reproductive structures in different stages of development. Scale $27 \mu \mathrm{m}, \mathrm{d}$ ) mature plurilocular sporangia (arrow head) and biseriate filaments (arrows) on the surface of the thallus. Scale $30 \mu \mathrm{m}$ 
Ectocarpus gonodioides Setchell \& N.L.Gardner (Fig. $3 \mathrm{a}, \mathrm{b}, \mathrm{c}, \mathrm{d})$

Talo microscópico, endófito y formando pequeños agregados de 500-600 $\mu \mathrm{m}$ de alto, adheridos por largos filamentos rizoidales, hialinos que penetran en el hospedero; los filamentos que sobresalen con ramificación alterna, irregular y escasa, adelgazados hacia el ápice. Las células de estos filamentos poseen varios plastos, de 11-14,4 $\mu \mathrm{m}$ de diámetro y 14-22 $\mu \mathrm{m}$ de longitud y ápices ligeramente adelgazados.

Reproducción: Plurangios fusiformes pedunculados, naciendo lateralmente en los filamentos de 20-32 $\mu \mathrm{m}$ de diámetro y 70-82 $\mu \mathrm{m}$ de longitud.

Hábitat: Semiendófito de Codium isabelae W.R.Taylor, en pozas intermareales e intermareal expuesto.

Distribución: EEUU (California), México (Golfo de California de Puerto Peñasco a Bahía de los Ángeles, Bahía Agua Verde a Cabo Pulmo, La Majahuita, El Zapote de Madero-La Mesa, Michoacán).
Ectocarpus taoniae Setchell \& N.L.Gardner (Fig. 4a, b, c, d)

Talo filamentoso, formando agregados de 1-1,5 mm de alto, epífito y adherido al hospedero por un sistema de filamentos reptantes superficiales, filamentos erguidos simples, raramente ramificados, gradualmente adelgazados hacia el ápice. Células de los filamentos postrados con varios plastos alargados de 5-7 $\mu \mathrm{m}$ de diámetro y 8-12 $\mu \mathrm{m}$ de longitud, las células de los filamentos erguidos de 7,5-14,5 $\mu \mathrm{m}$ de diámetro y $18,5-20 \mu \mathrm{m}$ de longitud.

Reproducción: Plurangios elipsoidales, dispuestos lateralmente en los filamentos o en los filamentos postrados, de 18-20 $\mu \mathrm{m}$ de diámetro y 55-62 $\mu \mathrm{m}$ de longitud.

Hábitat: Epífito de Padina crispata, intermareal expuesto.

Distribución: EEUU (California), México (San Juan de Alima, La Majahuita, Michoacán).
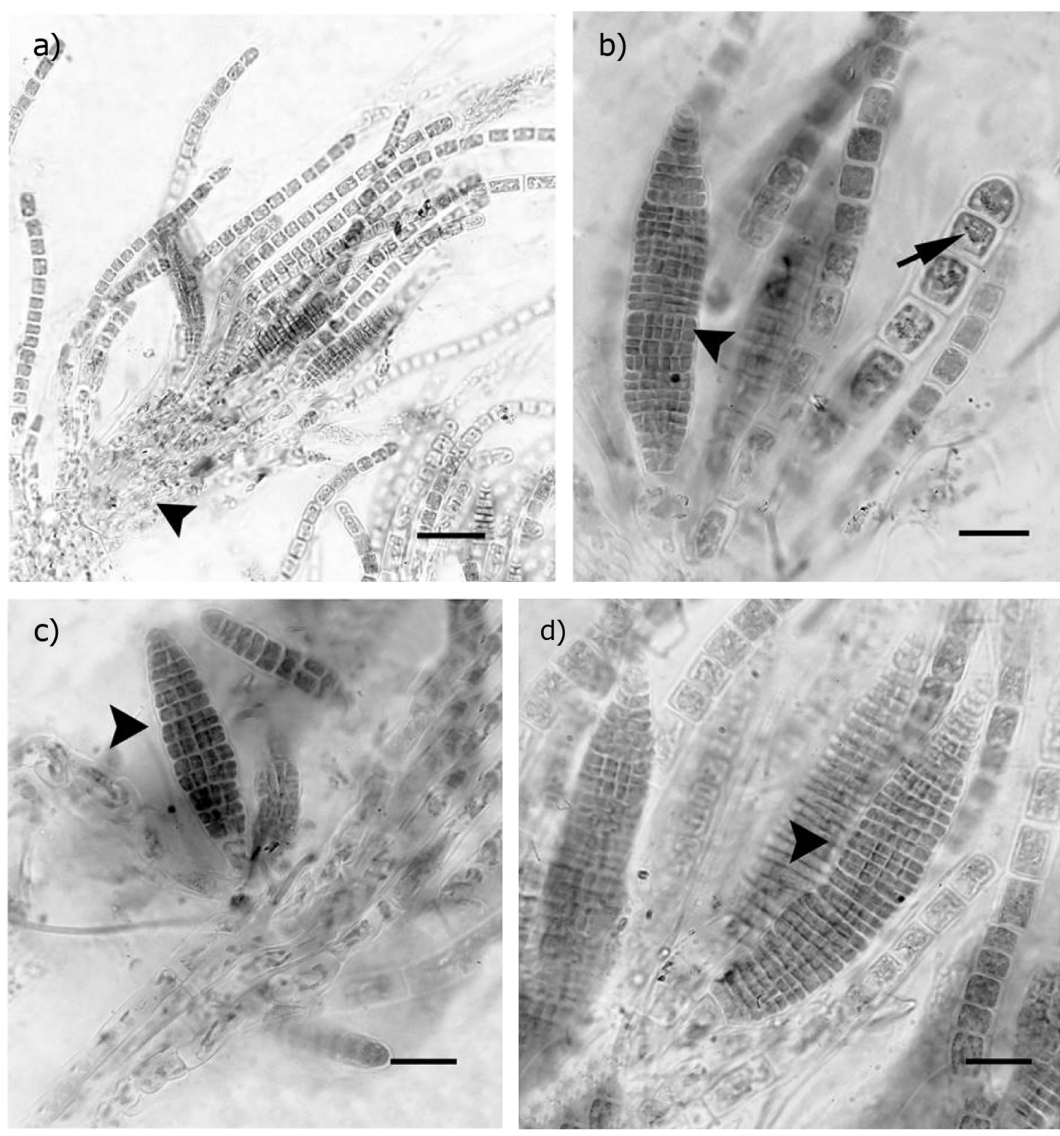

Figura 3. Ectocarpus gonodioides. a) Aspecto general del talo, y porción rizoidal que penetra al hospedero (cabeza de flecha). Escala $\mathbf{5 5} \mu \mathrm{m}, \mathrm{b}$ ) Plurangio (cabeza de flecha) y células que muestran el plasto en forma de banda (flecha). Escala $20 \mu \mathrm{m}, \mathrm{c}$ ) Plurangio terminal (cabeza de flecha). Escala $35 \mu \mathrm{m}$, d) Aspecto de los plurangios que emergen cerca de la porción rizoidal del talo (cabeza de flecha). Escala $\mathbf{2 6}$ um / Ectocarpus gonodioides. a) General aspect of the thallus, and rhizoidal portion that penetrates into the host (arrowhead). Scale $55 \mu \mathrm{m}$, b) Plurilocular sporangia (arrowhead) and cells showing the band-shaped plastids (arrow). Scale $20 \mu \mathrm{m}, \mathrm{c}$ ) Terminal plurilocular sporangia (arrowhead). Scale $35 \mu \mathrm{m}$, d) Aspect of plurilocular sporangia that emerge near the rhizoidal portion of the thallus (arrowhead). Scale $26 \mu \mathrm{m}$ 

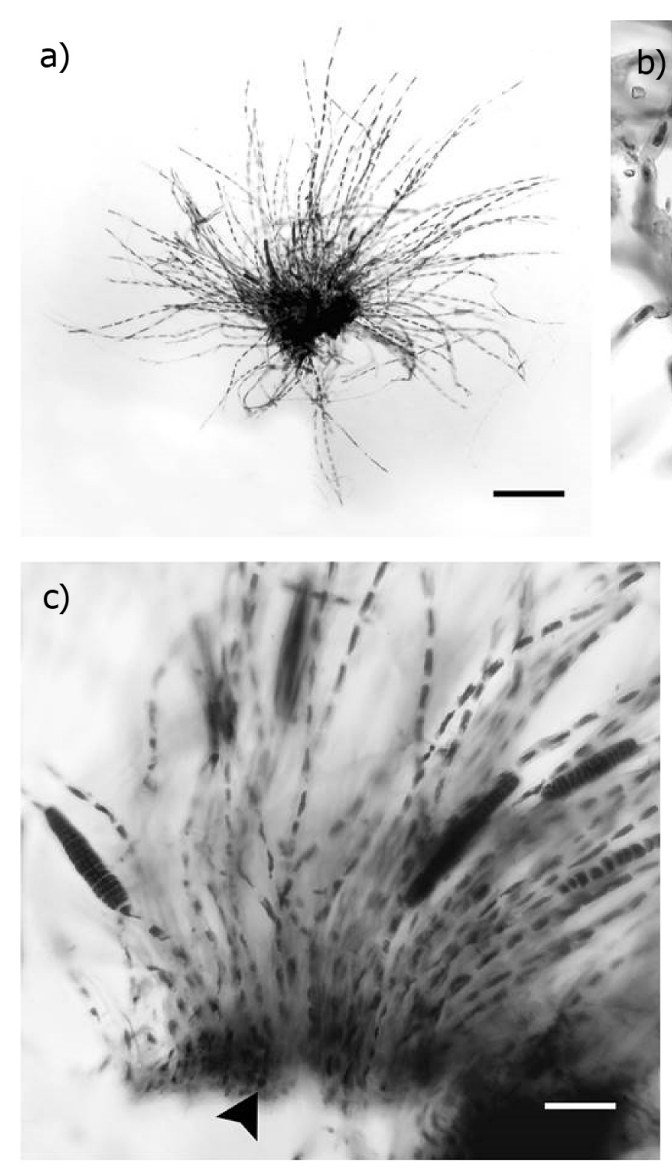
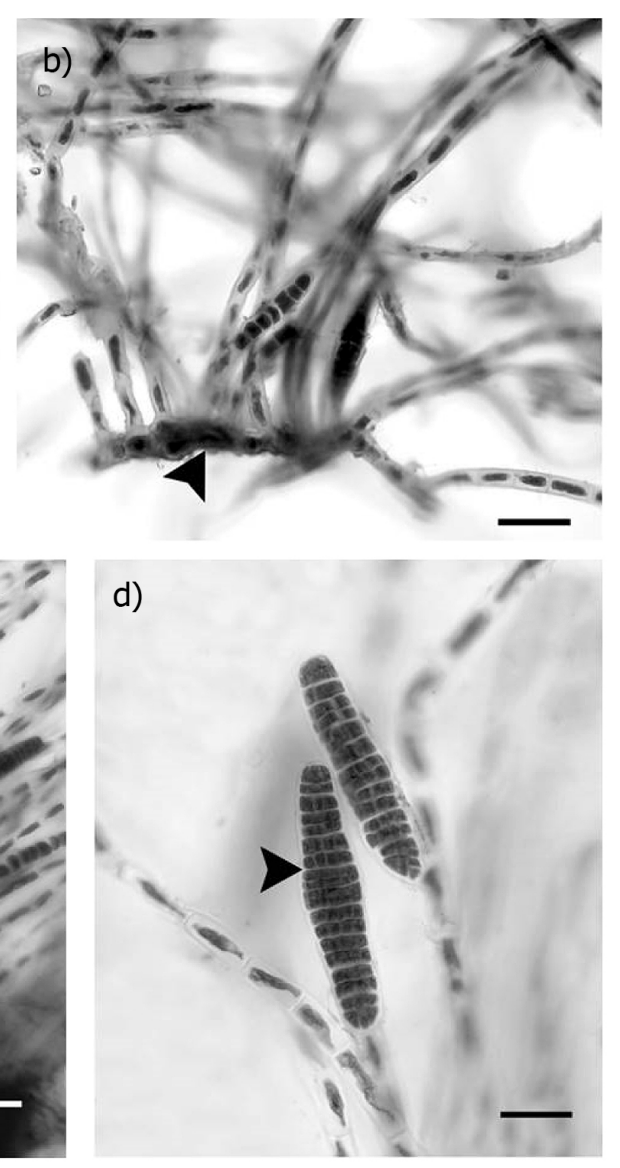

Figura 4. Ectocarpus taoniae. a) Aspecto general del talo filamentoso. Escala $475 \mu \mathrm{m}$, b) Porción de los filamentos postrados (cabeza de flecha) y los filamentos erguidos. Escala $32 \mu \mathrm{m}$, c) Porción basal densamente agregada (cabeza de flecha) y las porciones erguidas con plurangios. Escala $48 \mu \mathrm{m}, \mathrm{d}$ ) Plurangio terminal (cabeza de flecha) y lateral. Escala $22 \mu \mathrm{m}$ / Ectocarpus taoniae. a) General aspect of the filaments. Scale $475 \mu \mathrm{m}$, b) Portion of the prostrate filaments (arrowhead) and erect filaments. Scale $32 \mu \mathrm{m}$, c) Aspect of basal portion with entangled filaments (arrowhead) and erect filaments with plurilocular sporangia. Scale $48 \mu \mathrm{m}, \mathrm{d}$ ) Terminal plurilocular sporangia (arrowhead) and lateral plurilocular sporangia. Scale $22 \mu \mathrm{m}$

\section{Bachelotia antillarum (Grunow) Gerlof (Fig. 5a, b, c, d)}

Talos filamentosos, formando densos agregados de 9-10 mm alto, epilíticos y fijos al sustrato por filamentos rizoidales, los filamentos erguidos escasamente ramificados de forma alterna, células de los filamentos con uno o dos plastos estrellados de 35-40 $\mu \mathrm{m}$ de diámetro y 40-100 $\mu \mathrm{m}$ de longitud, ápices redondeados.

Reproducción: Plurangios intercalares 70-80 $\mu \mathrm{m}$ de diámetro, seriados.

Hábitat: Sobre rocas y epífita de otras macroalgas, intermareal expuesto.
Distribución: EEUU (Florida, Carolina del Norte, Hawái), Islas del Caribe (Bermuda, Barbados, Bahamas, Cuba), Guyana, Brasil, Europa (España, Italia, Portugal), África (Argelia, Angola, Gambia, Ghana, Marruecos, Nigeria, Senegal, Sierra Leona), India, Irán, Japón, Australia, Nueva Zelanda, Islas Salomón, México (Tabasco). Encontrada en este estudio en La Majahuita, El Zapote de Madero-La Mesa y Maruata-El Faro, Michoacán. 


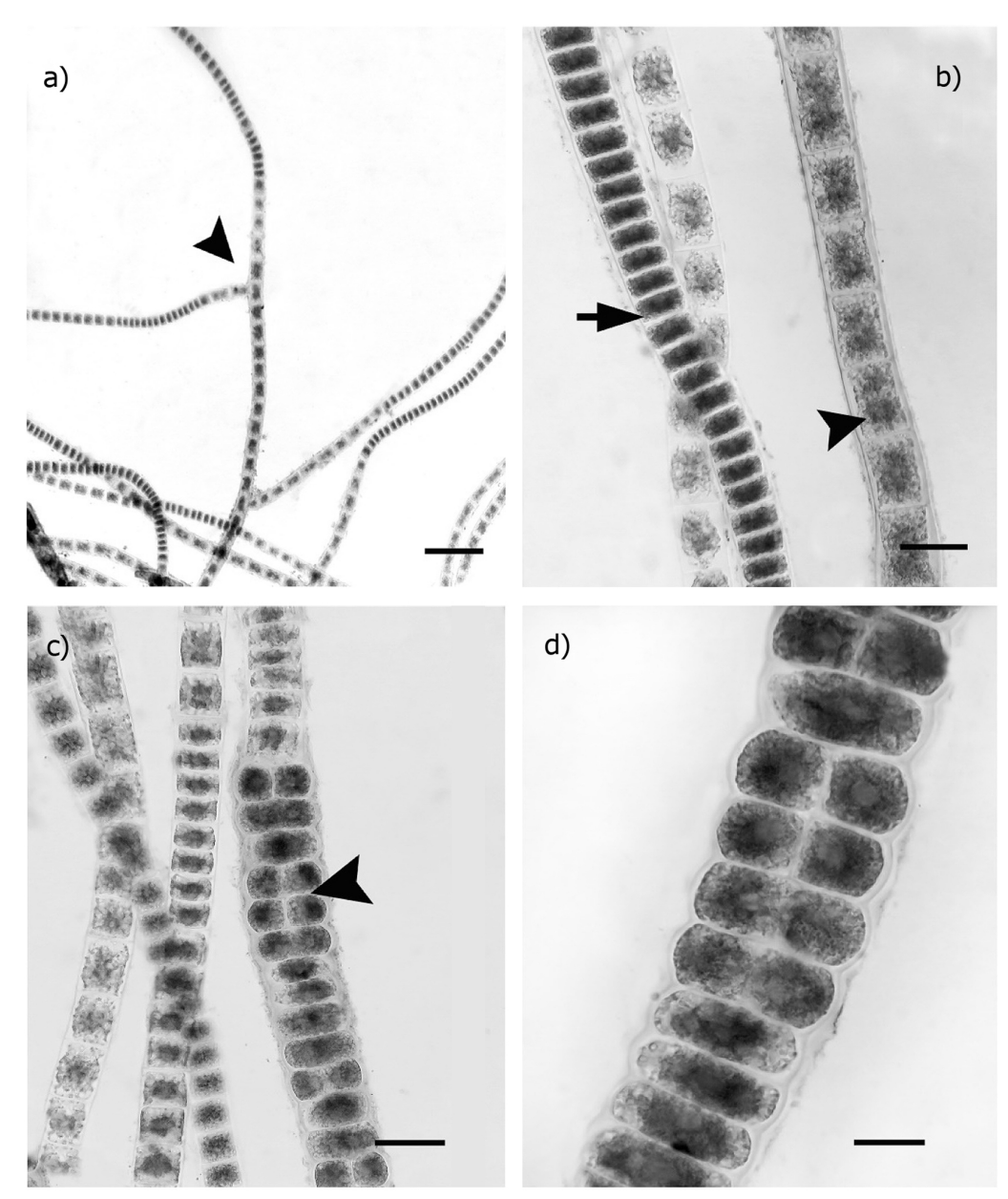

Figura 5. Bachelotia antillarum. a) Ramificación en las porciones apicales del talo (cabeza de flecha). Escala $150 \mu \mathrm{m}$, b) Filamentos, células con plasto lobado (cabeza de flecha) y plurangios intercalares jóvenes (flecha). Escala $41 \mu \mathrm{m}, \mathrm{c}$ ) Filamento en el que se observan las primeras divisiones periclinales de los plurangios (cabeza de flecha). Escala $\mathbf{6 1} \mu \mathrm{m}$, d) Filamento con plurangios intercalares. Escala $\mathbf{2 6} \boldsymbol{\mu \mathrm { m }}$ / Bachelotia antillarum: a) Thallus apical portion showing branching (arrowhead). Scale $150 \mu \mathrm{m}$, b) Cells showing lobate-plastids (arrowhead) and young intercalary plurilocular sporangia (arrow). Scale $41 \mu \mathrm{m}$, c) Filaments with young intercalary plurilocular sporangia (arrowhead). Scale $61 \mu \mathrm{m}$, d) Filaments with intercalary plurilocular sporangia. Scale $26 \mu \mathrm{m}$

\section{ESTADO REPRODUCTIVO}

Las algas en estado fértil analizadas en este trabajo fueron tanto esporofitos como gametofitos. Del total de taxones ubicados en este estudio, solo se encontraron 7 especies $(17,5 \%)$ en estado reproductivo sexual, presentando oogonios y/o anteridios. Los talos fértiles pertenecieron a Dictyota, Padina, Spatoglossum y Sargassum (Tabla 2). En el caso de Dictyota concrescens, D. crenulata, 4 especies de Padina y Spatoglossum subflabellatum, estas se encontraron con esporangios. También se encontraron a las especies de Sphacelaria con propágulos (multiplicación vegetativa). En el caso de Colpomenia, Rosenvingea, Ralfsia y Neoralfsia, se ubicaron en fase reproductiva con formación de zoosporas. Por lo que respecta a Feldmannia, Hincksia, Ectocarpus, Bachelotia, Compsonema, Hecatonema, Kuetzingiella, Streblonema y Asteronema presentan estructuras reproductivas llamadas unangios y/o plurangios, generalmente no es posible determinar si su contenido son esporas o gametos.

Por lo que respecta a Sphacelaria, los especímenes presentaron propágulos, lo anterior indica que $65 \%$ de especímenes se encontraron en reproducción asexual. Finalmente, el 17,5\% (7) de los taxones se halló en estado vegetativo (Tabla 2). 


\section{DiscUSIÓN}

El análisis del listado florístico permitió reconocer 27 registros infragenéricos nuevos para el litoral de Michoacán, 2 de ellos son nuevos para el Pacífico de México (Streblonema y Ectocarpus). De los 25 registros citados en la bibliografía, 13 taxones fueron encontrados en este estudio y 12 no se ubicaron en las muestras recolectadas (Tabla 2). Por lo tanto, el número de especies registradas para la costa de Michoacán se amplió a 52 taxones infragenéricos de algas pardas marinas bentónicas, incrementándose al doble de las especies totales de Phaeophyceae del área de estudio. La mayoría de los nuevos registros de algas pardas para Michoacán pertenecen a los géneros Dictyota, Padina, Spatoglossum, Compsonema, Bachelotia, Ectocarpus y Streblonema.

Se citan por primera vez para la costa del Pacífico de México y se describen a Streblonema anomalum y Ectocarpus taoniae (Tabla 2). También se describen dos especies, con pocas colectas en el Pacífico mexicano, Bachelotia antillarum y Ectocarpus gonodioides. Este último es un nuevo registro para el Pacífico tropical de México.

En general, las especies epífitas son desapercibidas por su talla pequeña, si están presentes en una sola estación del año y han sido poco estudiadas por los ficólogos. A continuación, se presentan algunos comentarios de las 4 especies descritas en este trabajo.

Las características de los ejemplares de Streblonema anomalum obtenidos en este estudio concuerdan en general con la descripción de Setchell \& Gardner (1922) para especímenes de California, EEUU. En la descripción original los autores indican que $S$. anomalum se encontró como endófito de Cumagloia andersonii (Farlow) Setchell $\&$ N.L.Gardner, mientras que en el área de estudio se ubicó en Dermonema virens. Tanto C. andersonii como D. virens pertenecen a la familia Liagoraceae. Su presencia en el área de estudio puede deberse a la corriente de California de aguas frías y cuya manifestación se presenta hacia el invierno y principios de primavera (SEMAR 2002). Esta descripción se considera una ampliación de sus límites de distribución.

En el caso de los especímenes asignados a Ectocarpus gonodioides, coinciden con lo descrito por Setchell \& Gardner (1922) para Isla Coronado en el Golfo de California, excepto en el diámetro de las células de los filamentos, que en nuestros ejemplares son más pequeñas. De acuerdo con Norris (2010), una característica distintiva de esta especie son los filamentos rizoidales que penetran profundamente en el hospedero Codium isabelae, lo que fue observado tanto en material de Michoacán como en especímenes del Golfo de California.
Por lo que respecta a Ectocarpus taoniae, las características de nuestros ejemplares se ajustan en general con la descripción de Setchell \& Gardner (1922) para especímenes de California, EEUU. Al igual que Streblonema anomalum, la presencia de E. taoniae en el área de estudio puede deberse a la corriente de California de aguas frías y cuya manifestación se presenta hacia el invierno y principios de primavera (SEMAR 2002). Lo anterior permite inferir que probablemente esta especie sea estacional y se desarrolle cuando la temperatura del agua de mar es baja. Además, puede pasar desapercibida por ser de talla pequeña y encontrarse epífita de otras especies de algas pardas como Padina crispata.

Finalmente, los especímenes asignados a Bachelotia antillarum concuerdan perfectamente con lo descrito por Taylor (1960). De acuerdo con este autor, las células en $B$. antillarum tienen plastos característicamente estrellados y plurangios intercalares, ambos observados en el material mexicano. Puede considerarse que esta especie tiene una distribución más amplia que lo registrado previamente. Anteriormente, esta especie solo tenía dos registros en Rocas Alijos, Baja California Sur y en la costa sur de Jalisco (Pedroche et al. 2008, Mendoza-González et al. 2011), por lo que el presente estudio representa el tercer registro de esta especie para el litoral del Pacífico de México. El número reducido de registros de esta especie en el Pacífico Tropical de México puede deberse a su hábito filamentoso y talla pequeña, por lo que puede pasar desapercibida para los recolectores.

El número total de taxones determinados fue más alto que lo registrado por Bastida-Zavala et al. (2013) para el litoral de Oaxaca, quienes señalan 38 taxones infragenéricos de algas pardas para esa región. La riqueza específica de algas pardas de Michoacán fue más alta que en la costa sur de Nayarit, donde Mateo-Cid \& MendozaGonzález (1992) citaron 16 taxones de Phaeophyceae. En 1991, las mismas autoras registraron 23 Phaeophyceae para el litoral de Colima. En Chiapas, Mendoza-González \& Mateo-Cid (1996) citan solo 7 taxones de Phaeophyceae en ese estado. Además, Mendoza-González et al. (2011) evidenciaron la presencia de 27 taxones de Phaeophyceae para el litoral sur de Jalisco.

Posteriormente, Mateo-Cid \& Mendoza-González (2012) registraron 25 taxones para la costa noroccidental de Guerrero. Lo anterior evidencia que el litoral de Michoacán tiene la mayor riqueza específica de algas pardas del litoral del Pacífico tropical mexicano, con numerosos taxones representativos de la región. De 25 registros previos, 12 no fueron ubicados en el presente estudio, y puede atribuirse a varios factores; en primer lugar sería la aplicación de nombres erróneos derivados de la falta de certeza de los caracteres taxonómicos actualmente en uso 
para la determinación de las especies, como por ejemplo, Montecinos et al. (2017) mencionan que la delineación precisa de las especies, los patrones de distribución y la diversidad para Ectocarpus siguen siendo problemáticos. Además, existe un elevado número de especies crípticas que requieren de estudios moleculares y una exhaustiva revisión taxonómica. Misma situación se presenta para Feldmannia y Hincksia (Kim 2010). En segundo lugar, las discrepancias en los registros pueden atribuirse a la realización de 15 recolectas en diferentes meses del año y este tipo de muestreo abarcó amplias zonas geográficas y nuevas localidades exploradas. Por último, los eventos climatológicos, como presencia de mar de fondo, lluvias atípicas y huracanes pueden inducir cambios en los ecosistemas marinos (Lugo 2000).

Se considera que el alto número de registros nuevos hallados en el área de estudio se debe al muestreo en localidades que no habían sido previamente estudiadas, la revisión meticulosa del material, que permitió ubicar especies epífitas y el mayor número de recolectas llevadas a cabo.

Entre los nuevos registros destacan por sus hábitos epífitos Dictyopteris delicatula, Feldmannia irregularis, F. simplex, Compsonema intricatum, Hecatonema streblonematoides y Kuetzingiella elachistaeformis. Es reducido el número de especies y estudios florísticos en que se cita a los representantes pequeños y filamentosos de la familia Chordariaceae (Pedroche et al. 2008), debido quizás a su hábito de vida epífito. Dentro del género Ectocarpus se encontró el mayor número de registros nuevos (4), sobresaliendo E. gonodioides y E. taoniae. En el caso de Padina y Sphacelaria, se ubicaron 3 y 2 registros nuevos, respectivamente (Tabla 2).

Por lo que respecta a los nuevos registros para el Pacífico de México, Streblonema anomalum y Ectocarpus taoniae, tienen distribución en mares templados. Anteriormente Dreckmann et al. (1990) y Stout \& Dreckmann (1993) indicaron que el litoral norte de Michoacán representa una zona de transición fitogeográfica en el gradiente ficoflorístico latitudinal entre las regiones tropical y templada del Pacífico. Lo anterior pudiera explicar la presencia de estas dos especies en el litoral de Michoacán.

La presencia de acantilados, plataformas rocosas, canales de corriente y agregados rocosos, combinado con características oceanográficas particulares, actúan en conjunto en el área de estudio y proveen las condiciones para el desarrollo de un elevado número de algas pardas (Dreckmann et al. 1990, Mateo-Cid \& Mendoza-González 1991, 2001, 2012; Stout \& Dreckmann 1993, MendozaGonzález et al. 2011, 2018). Igualmente, los resultados obtenidos indican que el litoral de Michoacán es un área con alta diversidad florística, si se tiene en cuenta que en su franja costera se pueden encontrar el $29,12 \%$ de las Phaeophyceae conocidas para la costa del Pacífico de México, que es de 182 especies (Pedroche et al. 2008). Es importante resaltar que numerosos taxones de Phaeophyceae de la costa del Pacífico tropical de México han sido recolectadas en sustrato rocoso. MateoCid \& Mendoza-González (1991, 2001, 2012), así como Mendoza-González et al. (2011) señalan que este sustrato expuesto al oleaje es el lugar donde se localiza una gran diversidad de algas marinas. La textura, el grado de dureza y el color de las rocas influyen sobre las comunidades algales. Por ejemplo, Dictyota concrescens, D. friabilis, Bachelotia antillarum y Sphacelaria tribuloides se pueden albergar entre los resquicios de las rocas y con ello se protegen del oleaje. En el caso de Padina crispata, Chnoospora minima y Sargassum liebmannii habitan en sustrato rocoso liso y muy expuesto al oleaje. En todos estos casos, las estructuras de adhesión tienen modificaciones relacionadas con la superficie utilizada.

Las diferencias en la riqueza específica entre las localidades de muestreo pueden ser explicadas por el tipo de sustrato dominante en los sitios de muestreo. Por ejemplo, en La Majahuita y El Zapote de MaderoLa Mesa, que son lugares con sustrato rocoso y grandes plataformas rocosas donde se forman pozas, lagunetas y canales, se ubicaron 27 y 21 taxones, respectivamente. Esto concuerda con Mendoza-González et al. (2011, 2018) y Mateo-Cid \& Mendoza-González (1992, 2001, 2012), quienes encontraron que las algas pardas que habitan en las costas mexicanas del Pacífico tropical de México se desarrollan principalmente sobre sustratos duros. Santelices (1977) indica que los sustratos duros y estables permiten la fijación y desarrollo de un mayor número de algas marinas bentónicas.

Por lo que respecta a San Telmo, Caleta de Campos y Tres Palmas-Playa Rangel, la riqueza específica fue baja comparada con las otras localidades $(2,4$ y 3 taxones, respectivamente). Esto puede atribuirse a que las playas son mayormente arenosas y con formaciones rocosas expuestas a intenso oleaje. En el caso de San Juan de Alima, Maruata-El Faro, La Saladita y Las Peñas se encontraron 7, 6, 8 y 7 taxones, respectivamente, a pesar de que en las localidades se encuentran formaciones rocosas. Dichos agregados rocosos se encuentran fuertemente expuestos al oleaje durante la resaca de las olas, en bajamar y pleamar.

Asimismo, se encontraron cambios de la riqueza específica a lo largo del periodo de estudio. La mayor riqueza se presentó en la época de secas (38 especies), que en México abarca de finales de invierno al inicio de primavera. Esto puede estar relacionado con la disponibilidad de luz. Lalli \& Parsons (1997) mencionan que en latitudes tropicales la superficie del agua de 
mar recibe más energía del sol a finales de primavera y durante el verano, los días en esta estación son 4 o $5 \mathrm{~h}$ más largos, lo que se traduce en mayor energía disponible para la fotosíntesis de las algas. En contraparte, el menor número de especies (19) se registró en la temporada de lluvias que abarca el final de verano y otoño. Durante este periodo la precipitación pluvial acarrea terrígenos al mar disminuyendo de este modo la energía proveniente del sol (Lalli \& Parsons 1997). Lo anterior concuerda con otros estudios realizados en otros estados del Pacífico sur de México. Por ejemplo, Mateo-Cid \& Mendoza-González (2012) en su estudio de algas marinas bentónicas de la costa noroccidental de Guerrero, encontraron un mayor número de especies de Phaeophyceae en la temporada de secas y lo atribuyeron en parte a las especies anuales, como Ectocarpus y Compsonema, que dominan en secas y disminuyen en lluvias. Se conoce que las variaciones temporales de la composición y riqueza de las algas marinas bentónicas es atribuible al efecto de la luz y la temperatura sobre las poblaciones de algas marinas, además de la precipitación, nutrientes, cambios en la dinámica del agua producto de los huracanes y tormentas (Santelices 1977).

En este estudio, se ubicó un alto número de especies de Phaeophyceae en estado reproductivo, hecho que coincide con lo registrado en diversos estudios florísticos realizados en las costas mexicanas (Mateo-Cid \& Mendoza-González 1991, 1992, 2001, 2012; Mendoza-González et al. 2000, 2011, Ávila-Ortiz \& Pedroche 2005). Estudios experimentales y observaciones de campo sugieren que la luz, la temperatura o la combinación de ambas, a menudo inducen la fertilidad en algas marinas (Liu et al. 2017). En relación con la fenología reproductiva de Dictyotales, la proporción y distribución temporal de los gametofitos generalmente se considera muy reducida (Phillips 1988). Montañés et al. (2006) mostraron que en poblaciones de Zonaria tournefortii de Tenerife, el máximo porcentaje de gametofitos se alcanzaba en otoño y era solo del $3,2 \%$ de los individuos de la población. Para Dictyota dichotoma, Tronholm et al. (2008) encontraron también una dominancia de esporofitos. Pero en algunas estaciones del año, los gametofitos podían constituir hasta el 30\% de las poblaciones. En este estudio solo 5 especies de Dictyotales estaban en fase de gametofito, mientras que en 9 especies se ubicaron esporofitos. Este hecho concuerda con lo citado en la literatura (Phillips 1988, Montañés et al. 2006).

Las algas de la clase Phaeophyceae determinadas en este trabajo, han sido registradas en las zonas tropicales rocosas del mundo, incluida la costa del Pacífico de América, el Océano Indico, o el Atlántico Tropical de América (Pedroche et al. 2008, Mateo-Cid et al. 2013). DíazMartín \& Espinoza-Ávalos (2000) en un estudio de las algas pardas de la península de Yucatán citan 35 especies. Mencionan que las familias Dictyotaceae, Sargassaceae y Ectocarpaceae son las mejores representadas en el área de estudio, habitando la mayoría de ellas en sustrato rocoso (28, 10 y 5 especies, respectivamente). En el presente estudio, la Familia Dictyotaceae fue la más representada con 20 taxones. Es conocido que la familia Dictyotaceae tiene una amplia distribución en las regiones tropicales y templadas del mundo (Pedroche et al. 2008, Mateo-Cid et al. 2019).

En conclusión, al integrar y analizar los estudios previos con los resultados obtenidos en el presente estudio, se constata la necesidad de realizar inventarios ficoflorísticos en regiones del litoral michoacano poco trabajados, así como actualizar la ficoflora en intervalos de tiempo regulares. Los resultados determinados en este estudio son la base para futuras investigaciones de tipo ecológico y de conservación, por lo que se recomienda continuar con muestreos en las localidades estudiadas para obtener datos de los cambios espaciales y temporales de las algas pardas que permitan dar seguimiento y reconocer posibles variaciones en las condiciones ambientales de la costa de Michoacán, México.

\section{Agradecimientos}

Al Instituto Politécnico Nacional (SIP-20180489 y SIP-20180491), por facilitar el apoyo económico, las instalaciones y equipo necesario para el desarrollo de este estudio. La primera y segunda autora agradecen la beca otorgada por la Comisión de Operación y Fomento de Actividades Académicas (COFAA). LEMC y ACMG reconocen los incentivos del programa EDI-IPN.

\section{LITERATURA CITADA}

Ávila-Ortiz A \& FF Pedroche. 2005. El género Padina (Dictyotaceae, Phaeophyceae) en la región tropical del Pacífico mexicano. Monografías Ficológicas 2: 139-171.

Bastida-Zavala JR, MS García-Madrigal, EF RosasAlquicira, RA López-Pérez, F Benítez-Villalobos, JF Meraz-Hernando, M Torres-Huerta, A MontoyaMárquez \& NA Barrientos-Luján. 2013. Marine and coastal biodiversity of Oaxaca, Mexico. Check List 9: 329-390.

Brand DD. 2013. Estudio costero del Suroccidente de México, 264 pp. Instituto de Geografía, UNAM y El Colegio de Michoacán A.C., Morelia.

Chávez-Barrera ML. 1980. Distribución del género Padina en las costas de México. Anales de la Escuela Nacional de Ciencias Biológicas México 23: 45-51.

Díaz-Martín MA \& J Espinoza-Avalos. 2000. Distribution of brown seaweeds (Phaeophyta) in the Yucatán Peninsula, Mexico. Bulletin of Marine Science 66: 279-289.

Dreckmann KM, FF Pedroche \& A Sentíes. 1990. Lista florística de las algas marinas bentónicas de la costa norte de Michoacán, México. Boletín de la Sociedad Botánica de México 50: 19-42. 
Fletcher RL. 1987. Seaweeds of the British Isles. Vol. 3. Fucophyceae (Phaeophyceae), Part 1: 1- 359. British Museum (Natural History), London.

García E. 1983. Modificaciones al sistema de clasificación climática de Köppen (para adaptarlo a las condiciones de la República Mexicana), 246 pp. Instituto de Geografía, Universidad Nacional Autónoma de México. México, D.F.

Guiry MD \& GM Guiry. 2019. AlgaeBase. World-wide electronic publication, National University of Ireland, Galway. <http://www.algaebase.org $>$

Kim HS. 2010. Ectocarpaceae, Acinetosporaceae, Chordariaceae. In: Kim H-S \& S-M Boo (eds). Algal flora of Korea. Volume 2, Number 1. Heterokontophyta: Phaeophyceae: Ectocarpales. Marine brown algae 1: 5-153. National Institute of Biological Resources, Incheon.

La Barre S, P Potin, C Leblanc \& L Delage. 2010. The halogenated metabolism of brown algae (Phaeophyta), its biological importance and its environmental significance. Marine Drugs 8: 988-1010.

Lalli CM \& TR Parsons. 1997. Biological oceanography. An introduction, 336 pp. Elsevier Butterworth-Heinemann, Burlington.

León-Álvarez D \& J González-González. 1993. Algas costrosas del Pacífico tropical. En: Salazar-Vallejo S \& NE González (eds). Biodiversidad marina y costera de México, pp. 456-474. CONABIO / CIQROO, Chetumal.

León-Tejera H, D Fragoso, D León-Álvarez, C Candelaria, E Serviere-Zaragoza \& J González-González. 1993. Characterization of tidal pool algae in the Mexican tropical Pacific coast. Hydrobiologia 260/261: 197-205.

Liu X, K Bogaert, AH Engelen, F Leliaert, MY Roleda \& O DeClerck. 2017. Seaweed reproductive biology: environmental and genetic controls. Botanica Marina 60: 89-108.

Lugo A. 2000. Effects and outcomes of Caribbean hurricanes in a climate change scenario. Science of the Total Environment 262: 243-251.

Mateo-Cid LE \& AC Mendoza-González. 1991. Algas marinas bénticas de la costa del estado de Colima, México. Acta Botánica Mexicana 13: 9-30.

Mateo-Cid LE \& AC Mendoza-González. 1992. Algas marinas bentónicas de la costa sur de Nayarit, México. Acta Botánica Mexicana 20: 13-28.

Mateo-Cid LE \& AC Mendoza-González. 2001. Algas marinas bentónicas de la costa de Oaxaca, México. Anales de la Escuela Nacional de Ciencias Biológicas México 47: 11-26.

Mateo-Cid LE \& AC Mendoza-González. 2012. Algas marinas bentónicas de la costa noroccidental de Guerrero, México. Revista Mexicana de Biodiversidad 83: 905-928.

Mateo-Cid LE, AC Mendoza-González, AG Ávila-Ortiz \& S Díaz-Martínez. 2013. Algas marinas bentónicas del litoral de Campeche, México. Acta Botánica Mexicana 104: 53-92.

Mateo-Cid LE, AC Mendoza-González \& CM HernándezCasas. 2019. Diversity of brown algae (Ochrophyta, Phaeophyceae) of Sian Ka' an reserve Biosphere, Mexican Caribbean. Pakistan Journal of Botany 51: 1-7.
Mendoza-González AC \& LE Mateo-Cid. 1996. Contribución al estudio de la ficoflora marina de la costa del estado de Chiapas, México. Polibotánica 2: 61-118.

Mendoza-González AC, LE Mateo-Cid, R Aguilar-Rosas \& LE Aguilar-Rosas. 2000. La familia Sphacelariaceae (Sphacelariales, Phaeophyta) en las costas de México. Polibotánica 11: 21-48.

Mendoza-González AC, LE Mateo-Cid \& C Galicia-García. 2011. Integración florística de las algas marinas de la costa sur de Jalisco, México. Revista Mexicana de Biodiversidad 82: $19-49$.

Mendoza-González AC, LE Mateo-Cid, R AlvaradoVillanueva, F Sotelo-Cuevas, JGA Ceballos-Corona \& AGA Garduño-Acosta. 2018. Nuevos registros y lista actualizada de las algas verdes (Chlorophyta) del litoral de Michoacán, México. Revista Mexicana de Biodiversidad 89: 971-985.

Montañés MÁ, M Sansón \& J Reyes. 2006. Vegetative and reproductive phenology of Zonaria tournefortii (Dictyotales, Phaeophyceae) in sublittoral populations off the Canary Islands. Botanica Marina 49(5/6): 406-416.

Montecinos AE, L Couceiro, AF Peters, A Desrut, M Valero \& ML Guillemin. 2017. Species delimitation and phylogeographic analyses in the Ectocarpus subgroup siliculosus (Ectocarpales, Phaeophyceae). Journal of Phycology 53(1): 17-31.

Norris JN. 2010. Marine algae of the northern Gulf of California: Chlorophyta and Phaeophyceae, 276 pp. Smithsonian Institution Scholarly Press, Washington DC.

Pedroche FF, PC Silva, LE Aguilar-Rosas, KM Dreckmann \& R Aguilar-Rosas. 2008. Catálogo de las algas marinas bentónicas del Pacífico de México. II. Phaeophycota, 146 pp. Universidad Autónoma Metropolitana, Universidad Autónoma de Baja California, University of California Berkeley, Mexicali/Berkeley.

Phillips JA. 1988. Reproduction in southern Australian species of the Dictyotales (Phaeophyta). Botanica Marina 31(5): 437-446.

Santelices B. 1977. Ecología de algas marinas bentónicas. Efectos de factores ambientales, $488 \mathrm{pp}$. Universidad Católica de Chile, Santiago.

Schneider CW \& RB Searles. 1991. Seaweeds of the southeastern United States, Cape Hatteras to Cape Cañaveral, 568 pp. Duke University Press, Durham.

SEMAR. 2002. Atlas de dinámica costera de la República Mexicana, 220 pp. Dirección General de Investigación y Desarrollo, Dirección General Adjunta de Hidrografía y Cartografía, Secretaría de Marina, Armada de México, Ciudad de México.

Setchell WA \& NL Gardner. 1922. Phycological contributions. VI. New species of Ectocarpus. University of California Publications in Botany 7: 403-426.

Setchell WA \& NL Gardner. 1924. New marine algae from the Gulf of California. Proceeding of the California Academy of Science Series 4(12): 695-949.

Stout I \& KM Dreckmann. 1993. Macroalgas bentónicas del faro de Bucerias, Michoacán, México. Anales del Instituto de Biología, Universidad Nacional Autónoma México, Serie Botánica 64: 1-23. 
Taylor WR. 1945. Pacific marine algae of the Allan Hancock Expeditions to the Galapagos Islands. Allan Hancock Pacific Expeditions 12: 1-528.

Taylor WR. 1960. Marine algae of the eastern tropical and subtropical coasts of the America, $870 \mathrm{pp}$. University of Michigan Press, Ann Arbor.

Thiers B. 2017. Index Herbariorum: a global directory of public herbaria and associated staff. New York Botanical Garden's Virtual Herbarium. <http://sweetgum.nybg.org/science/ih/>
Tronholm A, M Sansón, J Afonso-Carrillo \& O De Clerck. 2008. Distinctive morphological features, life cycle phases and seasonal variations in subtropical populations of Dictyota dichotoma (Dictyotales, Phaeophyceae). Botanica Marina 51(2): 132-144.

Wynne MJ. 2017. A checklist of benthic marine algae of the tropical and subtropical western Atlantic: fourth revision. Nova Hedwigia Beihefte 145: 1-202.

Recibido el 30 de julio de 2019 y aceptado el 14 de agosto de 2020

Editor asociado: Pilar Muñoz M. 